\title{
Competencias PROFESIONALES DEMANDADAS EN EL MERCADO LABORAL PERUANO
}

\author{
Herberth Eduardo Gutiérrez Villaverde \\ Ángel Alejandro Agüero Correa \\ Escuela de Negocios de la Universidad de Lima. Lima, Perú
}

Recibido: 2 de setiembre de 2015 / Aprobado: 18 de setiembre de 2015

\begin{abstract}
Resumen
El presente trabajo describe las competencias más requeridas en los profesionales de diversas carreras por parte de las principales empresas establecidas en nuestro país, y que solicitan personal a través del suplemento Aptitus del diario El Comercio, así como de otros medios de comunicación escritos. A partir de la creación de una base de datos, en la que se almacenó más de 5700 anuncios de empleos correspondientes a los años 2012 y 2013, y mediante la aplicación de técnicas multivariadas, se caracterizó el mercado de empleos y se definieron las competencias de mayor demanda en las carreras profesionales.
\end{abstract}

Palabras clave: habilidades / competencias / minería de datos

\section{Professional Skills Demanded by the Peruvian Labour Market}

\section{Summary}

This paper describes the skills most required among professionals within various career paths by leading companies in the Peruvian market, which advertise staffing positions via the weekly magazine Aptitus, part of the newspaper El Comercio, and through other media outlets. This database was created by accumulating results from more than 5,700 job advertisements between 2012 and 2013. By applying multivariate techniques, the job market was identified and the most demanded competencies within professional careers were defined.

Key words: skills / competencies / data mining 


\section{Introducción}

El contenido de este artículo está basado en la investigación "Una aproximación a las características de la demanda laboral de profesionales, egresados y practicantes de las carreras de la Escuela de Negocios de la Universidad de Lima", por ello cabe resaltar que la fuente de las tablas y figuras presentadas es el trabajo mencionado.

Desde la década de 1970 se ha desarrollado una gran cantidad de investigaciones que confirman la tesis de McClelland (1973), la cual afirma que para determinar quiénes pueden lograr resultados sobresalientes en un determinado puesto de trabajo no bastan las pruebas de inteligencia (IQ); se requiere la medición de las "competencias" del candidato.

El eje del estudio ha sido la construcción de una base de datos de anuncios de empleos, principalmente del semanario Aptitus del diario El Comercio, del período abril de 2012 - marzo de 2013. En dichos avisos, las empresas del mercado hacen referencia a las capacidades o competencias buscadas en el puesto ofrecido.

Esta información posibilitó caracterizar la demanda de empleos y extraer la valoración de las competencias por las empresas en general. De igual manera, se han efectuado asociaciones entre las competencias específicas requeridas con las especialidades buscadas para los puestos de trabajo.

\section{Revisión de la literatura}

Históricamente, las evaluaciones por competencias se utilizaron desde la antigüedad. Así, por ejemplo, en el siglo III de nuestra era, para ser aceptados como soldados en el ejército romano se necesitaba demostrar la capacidad de marchar dieciocho millas con armaduras, armas y sesenta libras de pertrechos (Singh, 2008a). En el siglo VII, el gobierno chino contrataba a los empleados públicos basado en pruebas de dominio de las obras de Confucio para poder emitir juicios basados en la interpretación de la filosofía de dicho autor (Singh, 2008b). En la época medieval, entre los siglos $V$ al XV, un aprendiz era reconocido como "competente" cuando sus productos podían ser aceptados de acuerdo a los estándares del comercio de la época (Hoge, Tondora y Marrelli, 2005).

Con el aporte pionero de Frederick Taylor, a inicios del siglo XX, aparecen especificaciones funcionales de los puestos de trabajo, que pueden considerarse como la primera expresión de lo que hoy conocemos como competencia, desde la perspectiva funcional (Chiavenato, 1998). Esto fue posteriormente desarrollado en Inglaterra en la década de 1980 (Delamare-Le Deist y Winterton, 2005).

En la literatura moderna hay consenso respecto a que David McClelland (1973, 1998) inició la corriente de análisis del desempeño con base en competencias, 
desde la perspectiva conductual. En su investigación cuestiona el sistema, prevalente en la época, de evaluación basada en pruebas de inteligencia y rasgos personales, debido a su ineficacia para capturar, fuera de contextos educacionales, la eficiencia o performance en un determinado puesto de trabajo.

A pesar del cúmulo de investigaciones efectuadas hasta la fecha, no hay una definición universal para el término "competencia" (Stevens, 2012; Delamare-Le Deist y Winterton, 2005); sin embargo, hemos considerado pertinente la siguiente definición conductista:

Un conjunto relacionado de conocimientos, capacidades y actitudes que afectan la mayor parte del trabajo de un individuo (un rol o responsabilidad) que está correlacionada con la performance en el trabajo, que puede ser medida a través de estándares aceptados y que puede ser mejorado por medio de entrenamiento y desarrollo. (Lucia y Lepsinger, 1999, p. 5)

La relevancia de esta definición está en la consideración de todas las variables como conocimientos, capacidades y actitudes - no solo la inteligencia- para establecer lo que sería un "desempeño superior" en el puesto. Del mismo modo, se correlacionarían estas competencias con el desempeño en el puesto de trabajo, medido bajo estándares aceptados. Por último, estas competencias pueden ser sujetas de medición y, por lo tanto, de desarrollo en los individuos que no la poseen, o la poseen en grado menor.

Otra definición bastante aceptada en la literatura es la de Spencer y Spencer (1993), que establece que competencia "es una característica subyacente de un individuo que está causalmente relacionada a un estándar de efectividad y/o con un desempeño superior en un trabajo o situación" (p. 9). En esta definición se supone que la competencia es un componente central de la personalidad y que por lo tanto puede explicar el comportamiento del individuo en un contexto laboral, indicando además si el desempeño será superior con respecto a un estándar predefinido.

De acuerdo con la European Credit Transfer and Accumulation System (2015), en los últimos años muchos gobiernos han mostrado preocupación por acercar los sistemas educativos a la formación basada en competencias con dos fines primordiales: evidenciar el valor de trabajadores que han alcanzado ciertos niveles de competencias que requieren las empresas, pero no a través de sistemas formales de educación; y, además, para acercar a los profesionales con educación formal a las demandas del mercado expresadas en competencias.

Como ejemplo se puede citar que el gobierno de Estados Unidos, a través de la Academia Nacional de Ciencias (National Research Council, 2008, 2010, 2011), en los últimos diez años viene realizando una serie de talleres para investigar la evolución del mercado laboral y su proyección en los plazos largo y mediano, en 
relación con las competencias que serán requeridas en el futuro y a los impulsores de estas competencias.

Así, se han definido las llamadas competencias para el siglo XXI, que son grupos de competencias transversales a todos los puestos de trabajo agregadas en tres categorías: cognitivas, interpersonales e intrapersonales.

a) Grupo de competencias cognitivas. Comprende las capacidades para la resolución de problemas no rutinarios, los que no pueden mecanizarse en computadoras: pensamiento crítico y pensamiento sistémico.

b) Grupo de competencias interpersonales. También conocidas como social skills, son las habilidades requeridas para interrelacionarse con las otras personas: comunicaciones complejas, capacidades sociales, trabajo en equipo, sensibilidad cultural y manejo de la diversidad.

c) Competencias intrapersonales. Son los talentos y capacidades que residen dentro del individuo para permitirle alcanzar objetivos y resolver problemas: autogestión personal, administración del tiempo, autodesarrollo, autorregulación, adaptabilidad y funcionamiento ejecutivo.

Por otro lado, para las universidades debería ser relevante preguntarse si dichas instituciones educativas toman en cuenta las competencias catalogadas como importantes por las empresas del mercado.

En un reciente estudio, Becerra y La Serna (2010) muestran que las tendencias globales observadas en los procesos de selección y reclutamiento se basan en competencias y son práctica común en las organizaciones especializadas en Recursos Humanos en el Perú. Este mismo estudio hace notar que no hay un alineamiento en lo requerido por las empresas y lo brindado por las universidades consideradas en el estudio, para lo cual recomienda acciones con la finalidad de ajustar los procesos formativos de los egresados.

Esta misma situación de no alineación entre los procesos de formación de egresados de pregrado y posgrado (Master in Business Administration - MBA) también ha sido observada en Estados Unidos y Canadá.

En el caso de pregrado, Abraham y Karns (2009), a partir de 42 universidades de Estados Unidos y Canadá con pregrados en administración y acreditadas por la Association to Advance Collegiate Schools of Business (AACSB), encontraron que si bien las autoridades de las universidades estaban de acuerdo con los ejecutivos de las empresas sobre cuáles eran las competencias requeridas para el éxito en los puestos de trabajo, dentro de los programas educativos no se enfatizaba en la formación de las competencias percibidas como importantes.

En lo concerniente a posgrado, Rubin y Dierdorff (2009) estudiaron 373 escuelas de negocios con programas MBA acreditadas en la AACSB, y encontraron que 
en sus respectivos currículos estas enfatizan más las categorías de competencias 'Gestión de la administración y control' y 'Gestión de la logística y la tecnología', mientras que los gerentes del mercado valoran más las categorías de 'Gestión de procesos para la toma de decisiones' y 'Gestión del capital humano'.

Estos hallazgos importantes muestran que no hay un alineamiento estratégico entre las competencias impartidas en las universidades y las competencias consideradas importantes para las empresas.

\section{Metodología}

El elemento de análisis es el anuncio en el que se solicita un candidato, por lo menos con estudios universitarios, con las siguientes variables: categoría de empleo ofrecido, puesto por cubrir, área de la empresa del puesto de trabajo, carrera o alternativas de carreras del candidato, competencias solicitadas, requisitos adicionales y experiencias profesionales requeridas, entre otras.

Las definiciones de competencias genéricas y específicas han sido tomadas del libro editado por Beneitone, Esquetini, González, Marty, Siufi y Wagenaar (2007), puesto que constituye el fruto del trabajo de 180 universidades latinoamericanas desde el año 2003.

Existen dos niveles de análisis de la base de datos: caracterización de la demanda de las carreras y caracterización de las competencias requeridas por el mercado.

\subsection{Caracterización de la demanda de carreras}

Se ha considerado la importancia relativa de cada carrera en la demanda por sectores público/privado, sectores económicos y áreas empresariales, y el grado de especialización o sustitución de las carreras en los puestos de trabajo por otras similares.

La sustitución de las carreras se calcula de dos maneras:

a) grado de sustitución global de una carrera

b) grado de sustitución de una carrera en los puestos en los que aparecen dos o más carreras

\subsubsection{Grado de sustitución global de una carrera}

Mide cómo otras carreras compiten en los puestos en los que también se demanda la carrera analizada e indica el número de carreras que pueden sustituirla en los puestos en los que aparece dicha carrera; es importante señalar que el número de menciones de la carrera que se analiza es equivalente al número de puestos en los que aparece tal carrera. Este indicador se calcula de la siguiente forma: 


\begin{tabular}{|clll|}
\hline $\begin{array}{c}\text { Grado de } \\
\text { sustitución global } \\
\text { carreras en los puestos } \\
\text { en los que aparece la } \\
\text { carrera analizada }\end{array}$ & $\begin{array}{l}\text { Número total de puestos } \\
\text { en los que aparece la } \\
\text { carrera analizada }\end{array}$
\end{tabular}

\subsubsection{Grado de sustitución de una carrera en los puestos en los que aparecen dos o más carreras}

En este caso se trata de determinar cómo otras carreras compiten con la carrera analizada, en puestos en los que se solicitan dos o más carreras. Este cálculo es más específico en la medición de la sustitución, ya que se circunscribe a los puestos en los que se solicitan dos o más carreras para ser cubiertos. Es decir, es el grado de sustitución de una carrera cuando compite con otras. Este indicador se calcula de la siguiente forma:

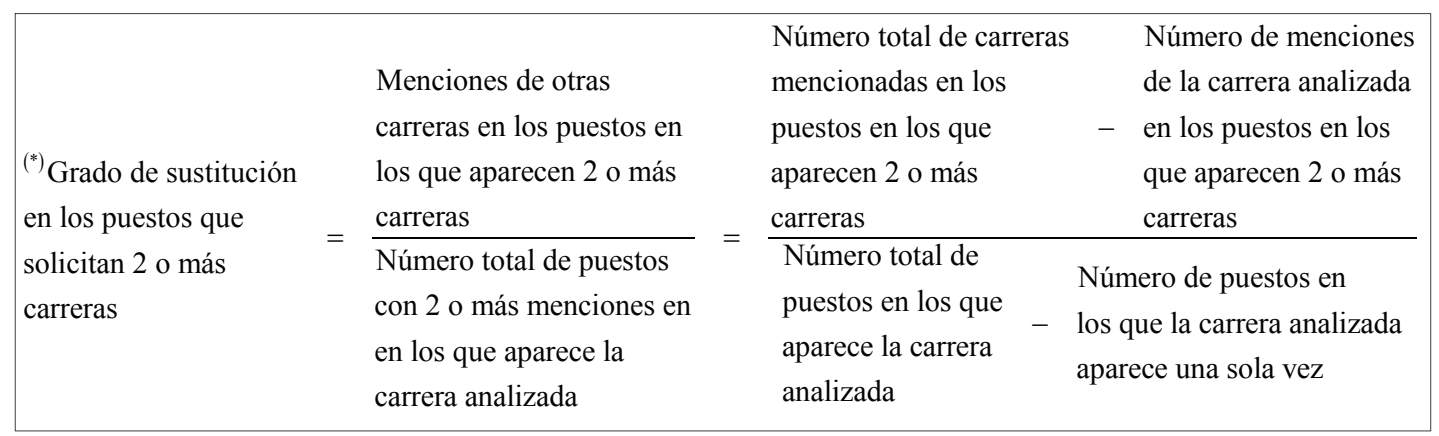

(*) Es el grado de sustitución en los puestos donde una carrera compite con otra, es decir, en los puestos donde se mencionan dos o más carreras, incluyendo a la que se analiza.

\subsection{Para la caracterización de las competencias}

Estas se registraron en la base de datos, considerando las expresiones de las competencias genéricas y específicas y haciendo una equivalencia con las del Tuning. Esta información fue analizada mediante el empleo de técnicas multivariantes, tales como el análisis de conglomerados y el análisis de correspondencias. 
3.

\section{Resultados}

\subsection{Caracterización de la demanda de las carreras}

En los 5700 anuncios en el periodo considerado se requirieron 6727 puestos de trabajo. En el $80 \%$ de los anuncios se solicitó solo un puesto y en el $20 \%$ restante solicitaron entre 2 y 69 posiciones de empleo. El $99 \%$ fueron para puestos de trabajo y $1 \%$ para practicantes. El $80 \%$ de los anuncios fue generado por empresas privadas, principalmente de los sectores industria y comercio, construcción, minería e hidrocarburos y otros servicios; y el $19 \%$ por empresas públicas, incluyendo las públicas/privadas de los sectores justicia, economía y finanzas, vivienda, construcción y saneamiento, y educación.

En la figura 1 se aprecia la distribución de los empleos solicitados por cada sector analizado.

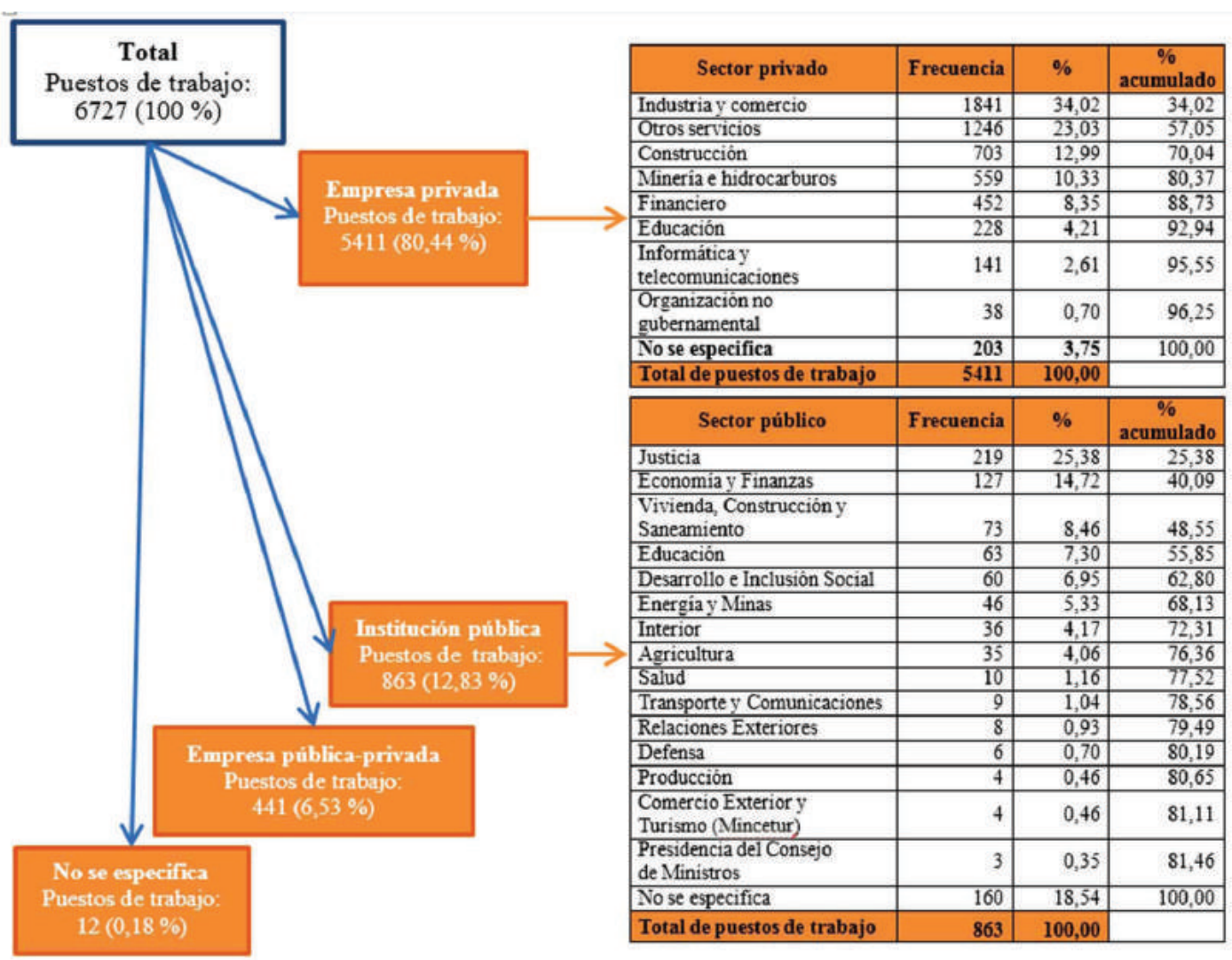

Figura 1. Empleos solicitados según sectores

Elaboración propia 
En la demanda de puestos de trabajo, aproximadamente solo el $6 \%$ de anuncios requiere estudios de posgrado además del bachillerato o del título profesional. El $52 \%$ requiere profesionales titulados; un $30 \%$ bachilleres y $13 \%$ egresados.

Entre las carreras más solicitadas destacan administración, contabilidad, ingeniería industrial, economía, derecho e ingeniería de sistemas, que representan el $70 \%$ de la demanda.

Para el $59 \%$ de los puestos, es decir 3945, se solicita una sola carrera (posiciones altamente especializadas); el resto requiere de hasta siete carreras (posiciones de alta sustitución entre carreras). El promedio general es de aproximadamente dos carreras por posición.

Las carreras más especializadas son las siguientes: contabilidad, administración, derecho, ingeniería industrial, ingeniería de sistemas, ingeniería civil, psicología, arquitectura, ingeniería de minas e ingeniería mecánica, que constituyen el 80 \% de las posiciones que solicitan una sola carrera.

En cuanto al grado de sustitución de las carreras se presentaron los siguientes resultados:

- En los puestos en los que se mencionan dos o más carreras, existen 2,19 menciones de otras carreras por una mención de la carrera analizada.

- La carrera de arquitectura tiene el menor grado de sustitución global, seguida por ingeniería civil.

- En lo concerniente a la sustitución global, arquitectura, ingeniería de sistemas, contabilidad, derecho, marketing, psicología y negocios internacionales tienen un grado bajo de sustitución. Administración, ingeniería industrial, economía y comunicaciones tienen un grado intermedio de sustitución global.

En lo que respecta a la relación entre la demanda de puestos de trabajo por sectores económicos y áreas empresariales, en la tabla 1 se muestra que la mayor cantidad de empleos pertenece al área empresarial de operaciones (39,92\%), de los sectores económicos industria y comercio, construcción y minería e hidrocarburos. En menor medida, el área empresarial de administración con el 17,75 \% de los puestos de trabajo, seguida por el área de finanzas, con el 14,93 \%. La mayoría de los puestos de administración corresponden a los sectores industria y comercio, y otros servicios. Los puestos del área de finanzas pertenecen mayoritariamente al sector financiero.

En lo que corresponde a los sectores, los más representativos son industria y comercio, minería e hidrocarburos, construcción, y otros servicios. Ello guarda relación con las tasas de crecimiento del PBI de estos sectores. 


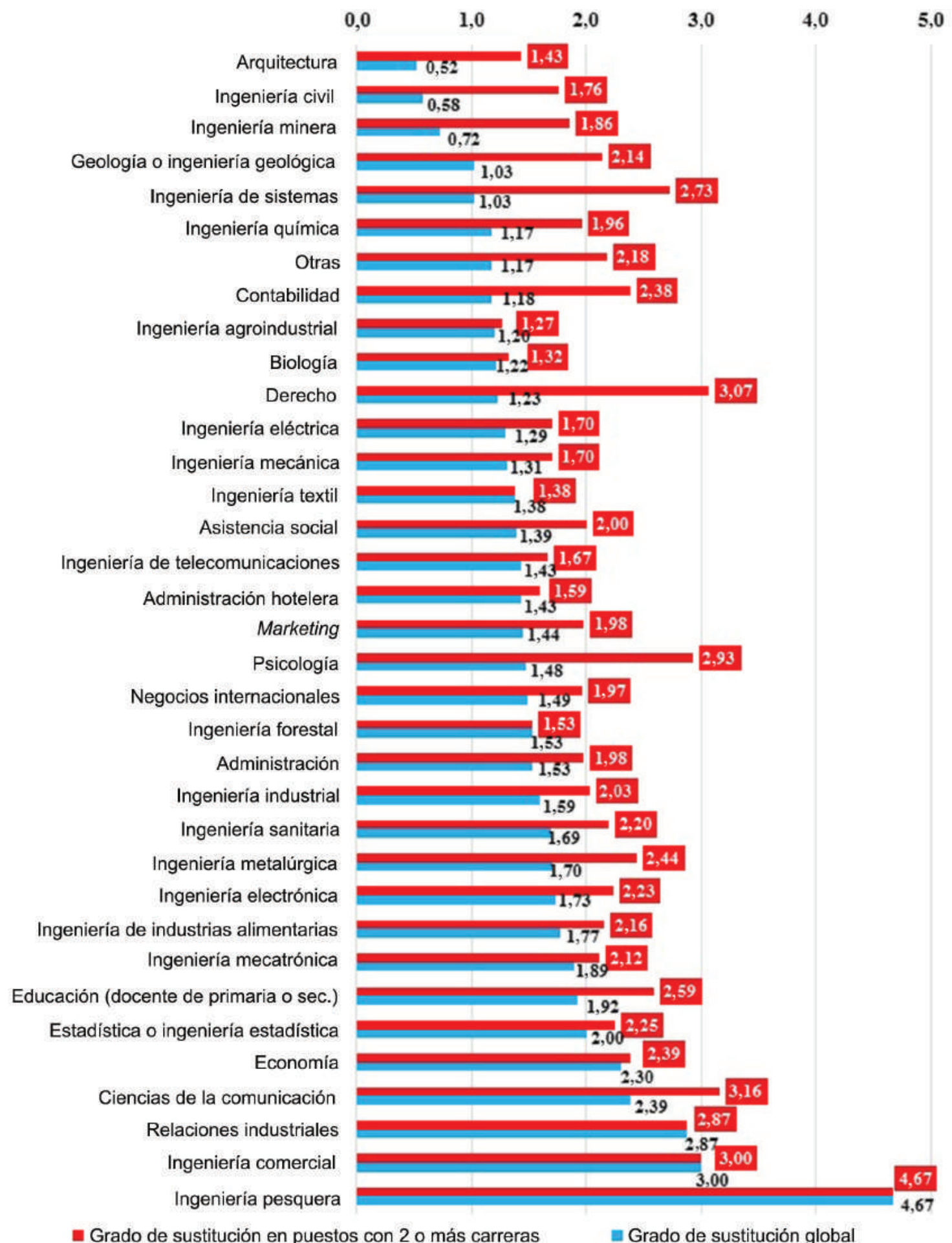

Figura 2. Grados de sustitución de las carreras

Elaboración propia 
El grado de intensidad de los enlaces (fuerte, medio y débil) se definen de la siguiente manera: primero se determina el rango de variación de las frecuencias observadas, el valor más alto del rango representa la asociación más fuerte encontrada entre los puestos de trabajo de un área empresarial (operaciones) y un sector económico (industria y comercio) y en forma análoga se define el de menor asociación. Luego se divide el rango en tres segmentos igualmente espaciados, donde el segmento más alto representa la asociación más fuerte, el segmento medio la asociación media y el segmento más bajo las asociaciones más débiles. Esta es una medida relativa del grado de asociación entre las variables área empresarial y sector económico (tabla 1).

Tabla 1

Puestos de trabajo por área empresarial según sector económico

\begin{tabular}{|c|c|c|c|c|c|c|c|c|c|c|}
\hline SectorlÄrea empresarial & $\begin{array}{c}\text { Operaciones } \\
\%\end{array}$ & $\begin{array}{c}\text { Administración } \\
\%\end{array}$ & $\begin{array}{c}\text { Finanzas } \\
\%\end{array}$ & $\begin{array}{c}\text { Comercial } \\
\%\end{array}$ & \begin{tabular}{|} 
Planificación \\
$\%$
\end{tabular} & $\begin{array}{c}\text { Legal } \\
\%\end{array}$ & $\begin{array}{c}\text { Sin írea } \\
\text { empresarial } \\
\text { especificada } \\
\%\end{array}$ & $\begin{array}{c}\text { Comunicaciones } \\
\%\end{array}$ & $\begin{array}{c}\text { Auditoria } \\
\%\end{array}$ & $\begin{array}{c}\text { Total } \\
\%\end{array}$ \\
\hline Industria y comercio & 14,75 & 7,42 & 4,85 & 10,90 & 2,63 & 0,91 & 0,09 & 0,24 & 0,18 & 41,97 \\
\hline Mineria e hidrocarburos & 9,66 & 2,18 & 0,48 & 0,39 & 0,94 & 0,36 & 0,24 & 0,12 & 0,03 & 14,42 \\
\hline Construcción & 9,87 & 1,27 & 0,64 & 0,36 & 0,91 & 0,27 & 0,42 & 0,00 & 0,00 & 13,75 \\
\hline Otros servicios & 2,70 & 3,69 & 2,48 & 1,73 & 0,39 & 0,91 & 0,18 & 0,21 & 0,42 & 12,72 \\
\hline Financiero & 1,15 & 1,51 & 5,97 & 0,70 & 0,33 & 0,21 & 0,00 & 0,06 & 0,21 & 10,15 \\
\hline Educación & 0,76 & 0,55 & 0,15 & 0,21 & 0,70 & 0,00 & 1,03 & 0,09 & 0,00 & 3,48 \\
\hline $\begin{array}{l}\text { Informática y } \\
\text { telecomunicaciones }\end{array}$ & 0,88 & 0,79 & 0,12 & 0,27 & 0,18 & 0,00 & 0,00 & 0,21 & 0,00 & 2,45 \\
\hline $\begin{array}{l}\text { Organización no } \\
\text { gubernamental }\end{array}$ & 0,15 & 0,33 & 0,24 & 0,09 & 0,06 & 0,09 & 0,03 & 0,06 & 0,00 & 1,06 \\
\hline $\begin{array}{l}\text { Total de carreras } \\
\text { solicitadas }\end{array}$ & 39,92 & 17,75 & 14,93 & 14,66 & 6,15 & 2,76 & 2,00 & 1,00 & 0,85 & 100,00 \\
\hline
\end{tabular}

\begin{tabular}{|l|l|}
\hline $\begin{array}{l}\text { Enlaces fuertes por } \\
\text { encima de } 9,83 \%\end{array}$ & \\
\hline Enlaces medios entre & \\
$9,83 \%$ y $4,92 \%$ & \\
\hline $\begin{array}{l}\text { Enlaces débiles por } \\
\text { debajo de } 4,92 \%\end{array}$ & \\
\hline
\end{tabular}

\section{Elaboración propia}

\subsection{Caracterización de las competencias requeridas en las carreras}

Las competencias genéricas exigidas por el mercado se presentan en la tabla 2, en la que se destacan: proactividad e iniciativa, trabajo en equipo, liderazgo, habilidades interpersonales, trabajo bajo presión, orientación al logro de objetivos y comunicación oral y escrita. 
Tabla 2

Competencias genéricas exigidas según área empresarial

\begin{tabular}{|c|c|c|c|c|c|c|c|c|c|c|}
\hline $\begin{array}{c}\text { Competencias generales / Áreas } \\
\text { empresariales }\end{array}$ & $\begin{array}{l}\text { Opera- } \\
\text { ciones } \\
\%\end{array}$ & $\begin{array}{c}\text { Adminis- } \\
\text { tración } \\
\%\end{array}$ & Comercial & $\mid \begin{array}{c}\text { Finanzas } \\
\%\end{array}$ & $\begin{array}{c}\text { Plani- } \\
\text { ficación } \\
\%\end{array}$ & $\underset{\%}{\text { Legal }}$ & $\begin{array}{c}\text { Comuni- } \\
\text { caciones } \\
\%\end{array}$ & \begin{tabular}{c|} 
Auditoría \\
$\%$
\end{tabular} & \begin{tabular}{|c|} 
Sin área \\
empresarial \\
identificada \\
$\%$ \\
\end{tabular} & $\begin{array}{c}\text { Total } \\
\%\end{array}$ \\
\hline Proactividad, iniciativa & 2,179 & 2,67 & 1,96 & 1,77 & 0,68 & 0,35 & 0,23 & 0,25 & 0,18 & 10,87 \\
\hline $\begin{array}{l}\text { Liderazgo, motivación y conducción hacia } \\
\text { metas comunes }\end{array}$ & 3,43 & 1,85 & 1,19 & 1,07 & 0,96 & 0,29 & 0,10 & 0,06 & 0,14 & 9,09 \\
\hline Trabajo en equipo & 2,67 & 2,11 & 1,25 & 1,25 & 1,07 & 0,14 & 0,12 & 0,23 & 0,14 & 8,98 \\
\hline Habilidades interpersonales & 1,72 & 1,93 & 1,25 & 1,01 & 0,76 & 0,39 & 0,14 & 0,06 & 0,12 & 7,38 \\
\hline Trabajo bajo presión & 2.59 & $\mathbf{1 , 5 0}$ & 1,33 & 0,86 & 0,33 & 0,08 & 0,06 & 0,25 & 0,02 & 7,02 \\
\hline Orientación al logro de objetivos & 1,99 & 0,96 & 1,19 & 0,82 & 0,31 & 0,04 & 0,02 & 0,18 & 0,02 & 5,53 \\
\hline Capacidad de negociación & 0,99 & 0,80 & 1,07 & 0,82 & 0,64 & 0,21 & 0,04 & 0,04 & 0,02 & 4,63 \\
\hline Creatividad, innovación & 1,09 & 1,19 & 0,51 & 0,57 & 0,66 & 0,18 & 0,06 & 0,06 & 0,14 & 4,46 \\
\hline Aplicación de conocimientos en la práctica & 0,66 & 0,86 & 0,45 & 0,64 & 0,51 & 0,27 & 0,14 & 0,02 & 0,06 & 3,61 \\
\hline $\begin{array}{l}\text { Coordinación, organización y planificación } \\
\text { del tiempo }\end{array}$ & 0,88 & 0,92 & 0,47 & 0,55 & 0,23 & 0,12 & 0,06 & 0,04 & 0,10 & 3,37 \\
\hline Solvencia moral, comportamiento ético & 0,62 & 0,94 & 0,53 & 0,70 & 0,06 & 0,35 & 0,10 & 0,00 & 0,08 & 3,38 \\
\hline Eficiencia & 0,80 & 0,84 & 0,39 & 0,45 & 0,02 & 0,10 & 0,10 & 0,02 & 0,02 & 2,74 \\
\hline Comunicación oral y escrita & 0,53 & 0,72 & 0,53 & 0,16 & 0,21 & 0,04 & 0,02 & 0,16 & 0,02 & 2,39 \\
\hline Orientación al cliente & 0,37 & 0,55 & 0,57 & 0,39 & 0,23 & 0,16 & 0,02 & 0,00 & 0,00 & 2,29 \\
\hline Capacidad crítica y autocrítica & 0,35 & 0,66 & 0,33 & 0,39 & 0,04 & 0,14 & 0,14 & 0,04 & 0,06 & 2,15 \\
\hline $\begin{array}{l}\text { Identificación, planteamiento y resolución de } \\
\text { problemas }\end{array}$ & 0,25 & 0,27 & 0,16 & 0,21 & 0,99 & 0,04 & 0,02 & 0,00 & 0,00 & 1,94 \\
\hline $\begin{array}{l}\text { Responsabilidad social y compromiso } \\
\text { ciudadano }\end{array}$ & 0,57 & 0,51 & 0,33 & 0,25 & 0,18 & 0,04 & 0,96 & 0,02 & 0,00 & 1,90 \\
\hline Toma de decisiones & 0,47 & 0,43 & 0,27 & 0,39 & 0,06 & 0,08 & 0,06 & 0,04 & 0,02 & 1,82 \\
\hline Vocación de servido & 0,16 & 0,60 & 0,37 & 0,23 & 0,02 & 0,14 & 0,04 & 0,02 & 0,02 & 1,60 \\
\hline Empatía & 0,08 & 0,31 & 0,23 & 0,23 & 0,55 & 0,02 & 0,00 & 0,04 & 0,00 & 1,46 \\
\hline Flexibilidad ante los cambios & 0,33 & 0,55 & 0,25 & 0,23 & 0,02 & 0,02 & 0,04 & 0,00 & 0,02 & 1,46 \\
\hline $\begin{array}{l}\text { Abstracción, pensamiento sistémico, análisis } \\
\text { y síntesis }\end{array}$ & 0,18 & 0,53 & 0,29 & 0,18 & 0,06 & 0,00 & 0,00 & 0,00 & 0,04 & 1,28 \\
\hline $\begin{array}{l}\text { Habilidades para buscar, procesar y analizar } \\
\text { información de fuentes diversas }\end{array}$ & 0,21 & 0,27 & 0,06 & 0,14 & 0,53 & 0,00 & 0,00 & 0,02 & 0,04 & 1,27 \\
\hline Adecuación a normas y procedimientos & 0,47 & 0,16 & 0,00 & 0,00 & 0,00 & 0,00 & 0,00 & 0,00 & 0,00 & 0,63 \\
\hline Compromiso con la calidad & 0,25 & 0,16 & 0,04 & 0,04 & 0,02 & 0,00 & 0,02 & 0,00 & 0,02 & 0,55 \\
\hline Tolerancia a la frustración & 0,08 & 0,16 & 0,14 & 0,06 & 0,04 & 0,04 & 0,00 & 0,00 & 0,00 & 0,52 \\
\hline Trabajo de campo & 0,16 & 0,10 & 0,02 & 0,12 & 0,00 & 0,06 & 0,02 & 0,02 & 0,02 & 0,52 \\
\hline Investigación & 0,16 & 0,18 & 0,06 & 0,02 & 0,02 & 0,06 & 0,00 & 0,00 & 0,00 & 0,50 \\
\hline Comunicación en otro(s) idioma(s) & 0,04 & 0,25 & 0,06 & 0,00 & 0,00 & 0,02 & 0,00 & 0,00 & 0,08 & 0,45 \\
\hline $\begin{array}{l}\text { Compromiso con la preservación del medio } \\
\text { ambiente }\end{array}$ & 0,04 & 0,18 & 0,06 & 0,04 & 0,02 & 0,00 & 0,02 & 0,00 & 0,06 & 0,42 \\
\hline $\begin{array}{l}\text { Conocimientos sobre el área de estudio y la } \\
\text { profesión }\end{array}$ & 0,06 & 0,06 & 0,06 & 0,02 & 0,00 & 0,02 & 0,00 & 0,02 & 0,00 & 0,24 \\
\hline Trabajar en forma autónoma & 0,02 & 0,04 & 0,00 & 0,04 & 0,00 & 0,00 & 0,00 & 0,00 & 0,02 & 0,12 \\
\hline Compromiso con su medio sociocultural & 0,00 & 0,00 & 0,04 & 0,00 & 0,00 & 0,00 & 0,00 & 0,00 & 0,00 & 0,04 \\
\hline $\begin{array}{l}\text { Aprendizaje y actualización } \\
\text { permanentemente }\end{array}$ & 0,02 & 0,00 & 0,00 & 0,00 & 0,00 & 0,00 & 0,00 & 0,00 & 0,00 & 0,02 \\
\hline $\begin{array}{l}\text { Total de competencias generales } \\
\text { solicitadas }\end{array}$ & 26,20 & 25,03 & 16,22 & 14,40 & 9,46 & 3,60 & 1,69 & 1,65 & 1,52 & 99,77 \\
\hline
\end{tabular}

Elaboración propia

En cuanto a las competencias genéricas por carreras, en la tabla 3 se han ordenado por filas las que más se solicitan en el mercado laboral, mientras que en las columnas el ordenamiento es por carreras, a través del orden descendente de frecuencia de las competencias. 
Tabla 3

Competencias genéricas solicitadas a las carreras en los puestos de trabajo

\begin{tabular}{|c|c|c|c|c|c|c|c|}
\hline Competencias genéricas/carreras & $\begin{array}{l}\text { Administración } \\
\%\end{array}$ & $\begin{array}{c}\text { Ingeniería } \\
\text { industrial } \\
\% \\
\end{array}$ & $\begin{array}{c}\text { Contabilidad } \\
\%\end{array}$ & $\begin{array}{c}\text { Economía } \\
\%\end{array}$ & $\begin{array}{c}\text { Derecho } \\
\%\end{array}$ & $\begin{array}{c}\text { Ingeniería } \\
\text { de sistemas } \\
\% \\
\end{array}$ & $\begin{array}{l}\text { Marketing } \\
\%\end{array}$ \\
\hline Proactividad, iniciativa & 3,04 & 1,78 & 2,05 & 1,00 & 0,60 & 0,40 & 0,35 \\
\hline Trabajo en equipo & 2,94 & 1,51 & 1,81 & 1,14 & 0,48 & 0,54 & 0,34 \\
\hline $\begin{array}{l}\text { Liderazgo, motivación y conducción hacia } \\
\text { metas comunes }\end{array}$ & 2,58 & 2,15 & 0,88 & 0,84 & 0,21 & 0,20 & 0,34 \\
\hline Habilidades interpersonales & 1,87 & 1,12 & 1,17 & 0,68 & 0,69 & 0,34 & 0,25 \\
\hline Trabajo bajo presión & 2,15 & 1,44 & 1,18 & 0,77 & 0,23 & 0,16 & 0,36 \\
\hline $\begin{array}{l}\text { Abstracción, pensamiento sistémico, análisis } \\
\text { y síntesis }\end{array}$ & 0,94 & 0,66 & 0,78 & 0,55 & 0,96 & 0,62 & 0,03 \\
\hline Orientación al logro de objetivos & 1,42 & 1,18 & 0,70 & 0,64 & 0,19 & 0,13 & 0,28 \\
\hline Capacidad de negociación & 1,21 & 0,88 & 0,39 & 0,52 & 0,11 & 0,09 & 0,20 \\
\hline Comunicación oral y escrita & 0,91 & 0,53 & 0,37 & 0,31 & 0,97 & 0,19 & 0,20 \\
\hline Creatividad, innovación & 0,91 & 0,66 & 0,31 & 0,31 & 0,07 & 0,18 & 0,10 \\
\hline $\begin{array}{l}\text { Coordinación, organización y planificación } \\
\text { del tiempo }\end{array}$ & 0,75 & 0,60 & 0,31 & 0,12 & 0,11 & 0,04 & 0,13 \\
\hline Solvencia moral, comportamiento ético & 0,64 & 0,43 & 0,46 & 0,22 & 0,16 & 0,11 & 0,10 \\
\hline Orientación al cliente & 0,64 & 0,34 & 0,25 & 0,24 & 0,17 & 0,07 & 0,13 \\
\hline Aplicación de conocimientos en la práctica & 0,62 & 0,52 & 0,30 & 0,24 & 0,12 & 0,09 & 0,09 \\
\hline $\begin{array}{l}\text { Identificación, planteamiento y resolución de } \\
\text { problemas }\end{array}$ & 0,61 & 0,34 & 0,29 & 0,40 & 0,04 & 0,02 & 0,05 \\
\hline Uso de TIC & 0,52 & 0,38 & 0,30 & 0,16 & 0,07 & 0,07 & 0,07 \\
\hline Eficiencia & 0,47 & 0,30 & 0,25 & 0,10 & 0,04 & 0,12 & 0,09 \\
\hline $\begin{array}{l}\text { Responsabilidad social y compromiso } \\
\text { ciudadano }\end{array}$ & 0,46 & 0,27 & 0,31 & 0,11 & 0,04 & 0,01 & 0,05 \\
\hline Empatía & 0,44 & 0,15 & 0,31 & 0,26 & 0,02 & 0,01 & 0,07 \\
\hline Capacidad crítica y autocrítica & 0,38 & 0,25 & 0,25 & 0,10 & 0,07 & 0,05 & 0,10 \\
\hline Toma de decisiones & 0,35 & 0,21 & 0,23 & 0,16 & 0,04 & 0,04 & 0,03 \\
\hline Vocación de servicio & 0,35 & 0,22 & 0,18 & 0,08 & 0,07 & 0,01 & 0,12 \\
\hline $\begin{array}{l}\text { Habilidades para buscar, procesar y analizar } \\
\text { información de fuentes diversas }\end{array}$ & 0,34 & 0,13 & 0,27 & 0,25 & 0,00 & 0,04 & 0,04 \\
\hline Flexibilidad ante los cambios & 0,38 & 0,20 & 0,12 & 0,07 & 0,02 & 0,03 & 0,03 \\
\hline Formulación y gestión de proyectos & 0,19 & 0,09 & 0,13 & 0,04 & 0,01 & 0,03 & 0,04 \\
\hline Trabajo de campo & 0,16 & 0,07 & 0,04 & 0,04 & 0,06 & 0,00 & 0,01 \\
\hline $\begin{array}{l}\text { Valoración y respeto por la diversidad y } \\
\text { multiculturalidad }\end{array}$ & 0,12 & 0,05 & 0,10 & 0,04 & 0,01 & 0,02 & 0,01 \\
\hline Adecuación a normas y procedimientos & 0,15 & 0,08 & 0,01 & 0,01 & 0,00 & 0,01 & 0,00 \\
\hline Compromiso con la calidad & 0,09 & 0,06 & 0,06 & 0,02 & 0,02 & 0,01 & 0,01 \\
\hline Tolerancia a la frustración & 0,11 & 0,08 & 0,05 & 0,02 & 0,02 & 0,00 & 0,01 \\
\hline Investigación & 0,12 & 0,07 & 0,02 & 0,01 & 0,02 & 0,03 & 0,01 \\
\hline $\begin{array}{l}\text { Conocimientos sobre el área de estudio y la } \\
\text { profesión }\end{array}$ & 0,06 & 0,04 & 0,05 & 0,04 & 0,01 & 0,01 & 0,01 \\
\hline $\begin{array}{l}\text { Compromiso con la preservación del medio } \\
\text { ambiente }\end{array}$ & 0,04 & 0,05 & 0,01 & 0,01 & 0,01 & 0,00 & 0,01 \\
\hline Comunicación en otro(s) idioma(s) & 0,04 & 0,03 & 0,01 & 0,04 & 0,01 & 0,07 & 0,01 \\
\hline Compromiso con su medio sociocultural & 0,01 & 0,00 & 0,00 & 0,00 & 0,00 & 0,00 & 0,01 \\
\hline Trabajar en forma autónoma & 0,01 & 0,02 & 0,01 & 0,00 & 0,00 & 0,00 & 0,00 \\
\hline Confidencialidad & 0,00 & 0,01 & 0,00 & 0,00 & 0,00 & 0,01 & 0,00 \\
\hline Aprendizaje y actualización permanente & 0,00 & 0,00 & 0,01 & 0,00 & 0,00 & 0,00 & 0,00 \\
\hline $\begin{array}{l}\text { Total de menciones de competencias } \\
\text { generales }\end{array}$ & 26,07 & $16.93 . \%$ & 13,98 & 9,57 & 5,69 & 3,76 & 3,73 \\
\hline
\end{tabular}

Enlaces fuertes por encima de $2,03 \%$

Enlaces medios entre 2,03\% y $1,02 \%$

Enlaces débiles por debajo de 1,02\%

\section{Elaboración propia}


Se observa que las principales competencias genéricas son las diez primeras que aparecen en la lista, ya que son requeridas con mayor frecuencia (tabla 4).

Tabla 4

\section{Competencias genéricas exigidas a los puestos que demandan una sola carrera}

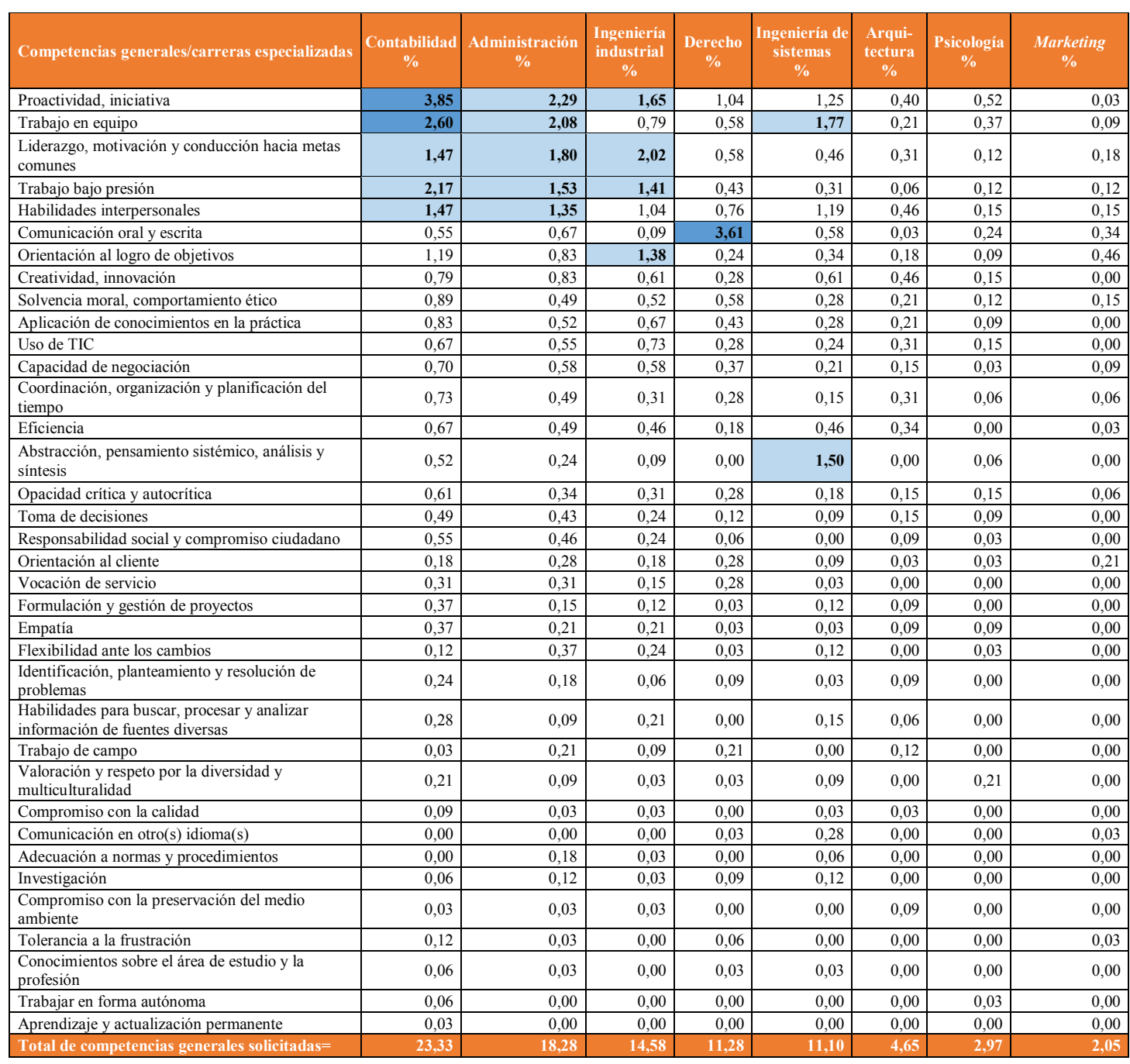

Enlaces fuertes por encima de $2,57 \%$

Enlaces medios entre $2,57 \%$ y $1,29 \%$

Enlaces débiles por debajo de 1,29\%

\section{Elaboración propia}


Las competencias específicas dependen de las disciplinas que conforman la carrera. En la tabla 5 se presentan las competencias específicas exigidas por el mercado. Como se aprecia, las que más destacan se relacionan con la gestión de negocios, desde las perspectivas financiera, contable, productiva y administrativa.

Tabla 5

\section{Competencias específicas más valoradas}

\begin{tabular}{|c|c|c|c|c|}
\hline & Competencias y conocimientos & Frecuencia & $\%$ & $\%$ acumulado \\
\hline 1 & Sistemas de costeo (ABC, estándar) & 136 & 5,47 & 5,47 \\
\hline 2 & Inteligencia de negocios & 128 & 5,15 & 10,62 \\
\hline 3 & Gestión de cobranzas & 126 & 5,07 & 15,69 \\
\hline 4 & Riesgo financiero & 124 & 4,99 & 20,68 \\
\hline 5 & Conocimiento de banca y finanzas & 121 & 4,87 & 25,55 \\
\hline 6 & $\begin{array}{l}\text { Análisis contable (cuentas por pagar, liquidaciones de } \\
\text { gastos, análisis de cuentas, conciliaciones bancarias, } \\
\text { liquidación) }\end{array}$ & 110 & 4,42 & 29,97 \\
\hline 7 & Análisis de EE. FF., planeación y estrategia financiera & 91 & 3,66 & 33,63 \\
\hline 8 & Conocimientos de equipos de plantas & 82 & 3,30 & 36,93 \\
\hline 9 & Legislación laboral & 78 & 3,14 & 40,07 \\
\hline 10 & Administración de recursos operativos y financieros & 76 & 3,06 & 43,13 \\
\hline 11 & Ofimática & 74 & 2,98 & 46,11 \\
\hline 12 & $\begin{array}{l}\text { Formulación, evaluación y dirección de proyectos de } \\
\text { inversión }\end{array}$ & 71 & 2,85 & 48,96 \\
\hline 13 & $\begin{array}{l}\text { Normas de gestión de calidad (ISO 9001:2000, ISO: } \\
\text { 14000, OSHA: 18000, ANSI, NIOSH, ASTM) }\end{array}$ & 68 & 2,73 & 51,69 \\
\hline 14 & Gestión de logística (compras, inventarios, almacenes) & 62 & 2,49 & 54,18 \\
\hline 15 & Finanzas & 62 & 2,49 & 56,67 \\
\hline 16 & Manejo de flujo de caja, efectivo & 57 & 2,29 & 58,96 \\
\hline 17 & Microfinanzas & 53 & 2,13 & 61,09 \\
\hline 18 & Sistemas y normas contables, financieras & 52 & 2,09 & 63,18 \\
\hline 19 & Contabilidad & 52 & 2,09 & 65,27 \\
\hline 20 & $\begin{array}{l}\text { Gestión de marketing (supervisar, diseñar e } \\
\text { implementar estrategias comerciales, nuevos productos) }\end{array}$ & 50 & 2,01 & 67,28 \\
\hline & Otros & 814 & 32,72 & 100,00 \\
\hline & Total & 2487 & 100,00 & \\
\hline
\end{tabular}

\section{Elaboración propia}




\section{Conclusiones}

El presente estudio aporta valiosos criterios para el alineamiento curricular, a través de los procesos de diseño curricular y formación académica en la educación superior. Por ejemplo, los conceptos de carreras especializadas o carreras con altos grados de sustitución, así como las competencias requeridas en cada una de ellas, se pueden usar para reorientar los temas y procedimientos considerados en la formulación o reformulación del perfil de la carrera.

Otro importante aporte es la información reportada acerca de la percepción sectorial de las empresas empleadoras sobre las competencias que consideran más útiles, de acuerdo con las áreas empresariales.

Se observa que las demandas laborales del mercado peruano siguen las tendencias globales en cuanto a expresarlas en términos de competencias. Esta situación se debe a que los procesos de reclutamiento y desarrollo de personal están basados, a diferencia de un pasado no muy lejano, en evidencias que ya no solamente miden los conocimientos y el coeficiente intelectual (IQ), sino que incluyen evaluaciones más integrales relacionadas directamente con la función que desempeñará el profesional que se va a contratar.

Por consiguiente, existe preocupación en las entidades educativas y formadoras de profesionales por comprender y caracterizar estas expresiones de la demanda en los mercados locales y globales, a fin de alinear sus procesos formativos y curriculares para responder a esos requerimientos más concretos.

La globalización es un impulsor de los enfoques basados en competencias, puesto que permite un lenguaje común para expresar las necesidades ocupacionales. Asimismo, genera una movilidad académica y laboral de dimensión global. Por esta razón, en los últimos años han surgido proyectos como el Tuning, primero en Europa y luego en Latinoamérica, cuya finalidad es posibilitar la movilidad laboral regional. De igual forma, las instituciones certificadoras de mayor prestigio en el ámbito global, como la AACSB, por mencionar una de ellas, emplea el enfoque de competencias en los procesos de acreditación de carreras y facultades.

\section{Referencias}

Abraham, S. E., y Karns, L. A. (2009). Do business schools value the competencies that businesses value? Journal of Education for Business, 84(6), 350-356. DOI: 10.3200/JOEB.84.6.350-356

Becerra, A. M., y La Serna, K. (2010). Las competencias que demanda el mercado laboral de los profesionales del campo económico-empresarial en la actualidad. Documento de discusión. Centro de Investigación de la Universidad 
del Pacífico. Recuperado de http://srvnetappseg.up.edu.pe/siswebciup/ Files/DD1005\%20-\%20Becerra_La\%20Serna.pdf

Beneitone, P., Esquetini, C., González, J., Marty, M., Siufi, G. y Wagenaar, R. (Eds.) (2007). Reflexiones y perspectivas de la educación superior en América Latina. Informe final - Proyecto Tuning - América Latina 2004-2007. Bilbao: Universidad de Deusto y Universidad de Groningen.

Chiavenato, I. (1998). Administración de recursos humanos. Bogotá: McGraw-Hill.

Delamare-Le Deist, F., y Winterton, J. (2005). What is competence? Human Resource Development International, 8(1), 27-46. DOI: 10.1080/1367886042000338227

European Credit Transfer and Accumulation System (ECTS). (2015). What is it? Recuperado de http://ec.europa.eu/ education/ects/ects_en.htm

Hoge, M. A., Tondora, J., y Marrelli, A. F. (2005). The fundamentals of workforce competency: implications for behavioral health. Administration and Policy in Mental Health and Mental Health Services Research, 32(5-6), 509-531. DOI: org/10.1007/s10488-005-3263-1

Lucia, A., y Lepsinger, R. (1999). The art and science of competency models: Pinpointing critical success factors in organizations. San Francisco, CA, Estados Unidos: Jossey-Bass/Pfeiffer.

McClelland, D. C. (1973). Testing for competence rather than for "intelligence". American Psychologist, 28(1), 1-14. DOI: 10.1037/h0034092

McClelland, D. C. (1998). Identifying competencies with Behavioral-Event interviews. Psychological Science, 9(5), 331-339. DOI: 10.1111/1467-9280.00065

National Research Council. (2008). Research on future skill demands: a workshop summary. Margaret Hilton, Rapporteur. Center for Education, Division of Behavioral and Social Sciences and Education. Washington, DC: The National Academies Press.

National Research Council. (2010). Exploring the intersection of science education and 21st century skills: a workshop summary. M. Hilton, Rapporteur. Board on Science Education, Center for Education, Division of Behavioral and Social Sciences and Education. Washington, DC: The National Academies Press.

National Research Council. (2011). Assessing 21st century skills: summary of a workshop. J. A. Koenig, Rapporteur. Committee on the Assessment of 21st Century Skills. Board on Testing and Assessment, Division of Behavioral and Social Sciences and Education. Washington, DC: The National Academies Press. The National Academies Press. 
Rubin, R. S., y Dierdorff, E. C. (2009). How relevant is the MBA? Assessing the alignment of required curricula and required managerial competencies. Academy of Management Learning \& Education, 8(2), 208-224.

Singh, R. (2008a). History of recruiting: Part I. Recuperado de http://www.eremedia. com/ere/history-of-recruiting-part-i/

Singh, R. (2008b). History of recruiting: Part II. Recuperado de https://www.eremedia. com/ere/history-of-recruiting-part-ii/

Spencer, L., y Spencer, S. (1993). Competence at work: models for superior performance. Nueva York: John Wiley \& Sons.

Stevens, G. W. (2012). A critical review of the science and practice of competency modeling. Human Resource Development Review, 12(1), 86-107. DOI: $10.1177 / 1534484312456690$ 
-
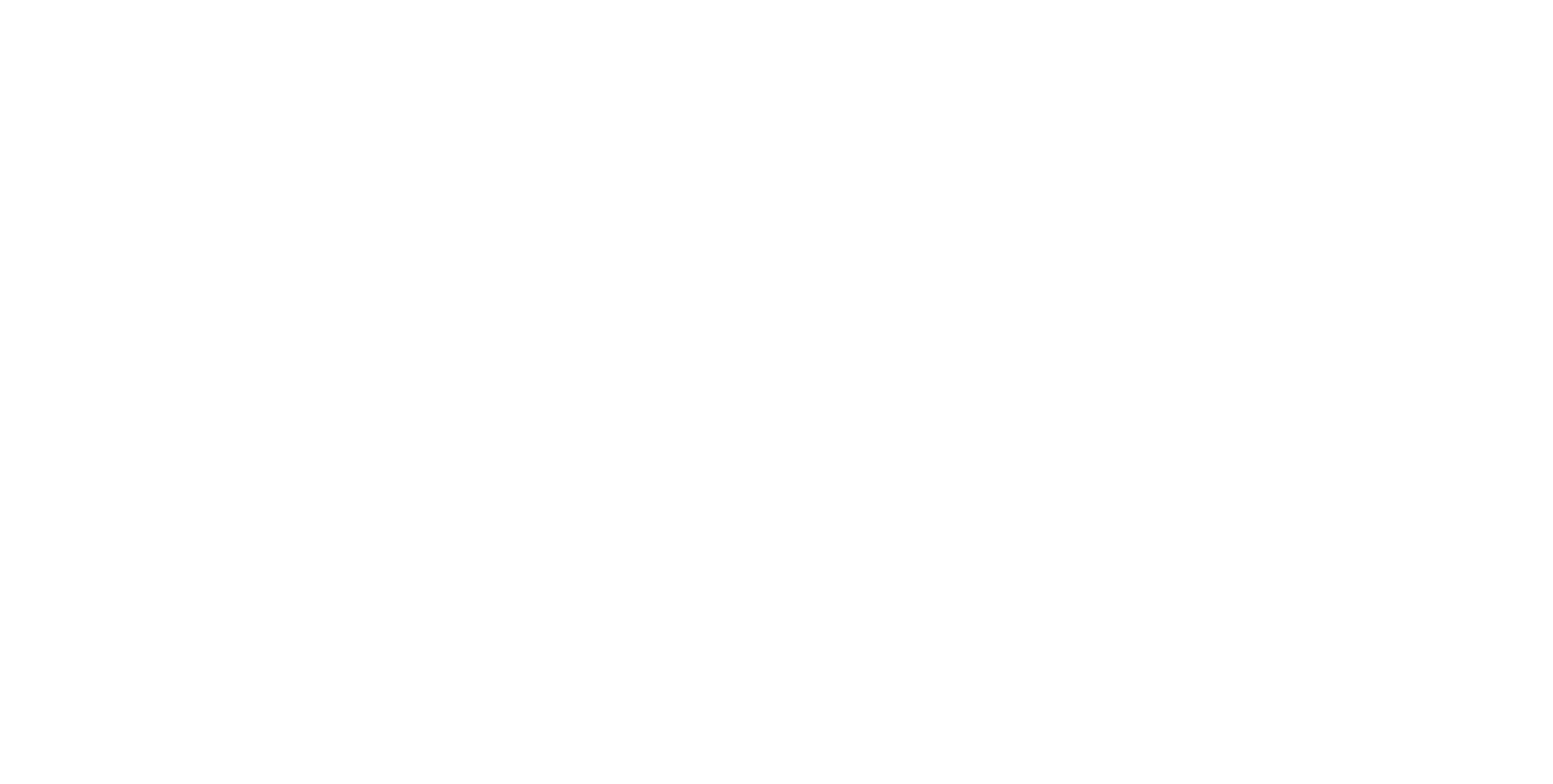